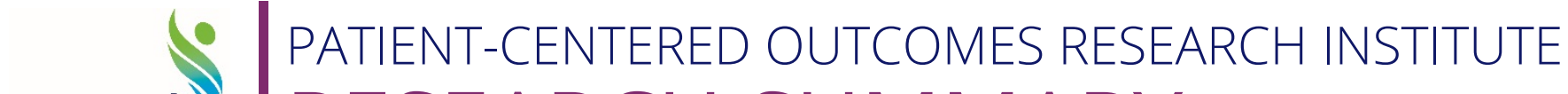 pcori?. RESEARCH SUMMARY
}

\section{Is a Patient Navigation Program More Helpful than a Referral Program for Reducing Depression and Improving Quality of Life among Women Living in Neighborhoods with Few Resources?}

Principal investigator Ellen Poleshuck, PhD
Organization University of Rochester

\section{What was the research about?}

When people are depressed, they feel sad, hopeless, or empty. Many people who live in neighborhoods with few resources have depression. They often face other life stressors, such as not having enough food or problems with relationships, personal safety, or housing.

In this study, the research team compared two programs designed to help women with feelings of depression who live in neighborhoods with few resources:

- Patient navigation program. In this program, the research team matched women with a mentor from the community. Mentors helped each woman decide which of her needs to focus on and supported her in getting help for those needs.

- Referral program. In this program, women received printed information about resources in the community. They also got help making appointments if needed. For example, the research team would offer to make an appointment with a therapist for a woman who reported mental health concerns.
The research team wanted to learn if women were more satisfied with one of the two programs. The team also wanted to know which program worked better to reduce feelings of depression and improve quality of life.

\section{What were the results?}

Women in both programs were very satisfied with their program. They liked the two programs about the same.

At the end of the study, feelings of depression and quality of life were similar for women in both programs. Both programs helped reduce feelings of depression. Overall, quality of life didn't change for women in either program. But among women who had depression plus other problems such as anxiety or pain, the patient navigation program improved some aspects of quality of life more than the referral program.

\section{Who was in the study?}

The study included 223 adult women who reported symptoms of depression and received care at three health clinics in Rochester, New York. Of these women, 57 percent were African American, 21 percent were white, and 19 percent were Hispanic. Also, 73 percent had a household income of less than $\$ 20,000$ per year, 
and 30 percent were pregnant when the study started. The average age was 30.

\section{What did the research team do?}

The research team assigned women by chance to either the patient navigation or referral program. Both programs lasted four months.

Women in the study completed questionnaires when the study began, when the programs ended, and then three and six months later. Questionnaires included questions about feelings of depression, quality of life, and how satisfied women in the study were with their program.

The team worked with an advisory group of patients, researchers, clinicians, policy makers, and others. The group gave feedback to help design and run the programs.

\section{What were the limits of the study?}

This study included women from three clinics in one city. Results might be different in other places. The programs lasted for four months. Four months may not have been long enough for quality of life to improve.

Future research could see if these two programs could help women with depression in other neighborhoods with few resources.

\section{How can people use the results?}

Health centers that serve women from neighborhoods with few resources may consider using either a patient navigation program or a referral program to help women with depression. Women with depression plus problems such as anxiety or pain may find a patient navigation program more helpful than a referral program.

To learn more about this project, visit pcori.org/Poleshuck096. 